deficiency to show that $12 / 15$-LO inhibitors could not only improve bone mass during development, but also offset the bone loss that is associated with oestrogen deficiency.

The authors suggest that these findings in mice could have relevance to human osteoporosis, especially as a region of human chromosome 17 that contains the genes encoding 12-LO and 15-LO has already been linked to low bone density. And if a role for the 12/15-LO pathway in regulating human bone mass is confirmed, inhibitors of this enzyme that are already under development for other indications, including atherosclerosis, could provide a good starting point for preventing the silent progression of osteoporosis in humans.

\section{Clare Ellis}

(0) References and links ORIGINAL RESEARCH PAPER Klein, R. F. et al. Regulation of bone mass in mice by the lipoxygenase gene Alox15. Science 303, 229-232 (2004)

FURTHER READING Goltzman, D. Discoveries, drugs and skeletal disorders. Nature Rev. Drug Discov. 1, 784-796 (2002)

\section{New forms of attack}

Treating sepsis - generally defined as the response of the host to microbial infection - is crucial in the clinical setting. Extreme forms of the condition, which usually arise as a result of complications from other diseases or treatments, has become the leading cause of death among patients in intensive care units. But developing therapies has proved difficult, as sepsis, although simple in definition, encompasses a wide range of inflammatory response mechanisms. Because of this complexity, two decades and billions of dollars invested in research on sepsis therapeutics has resulted in only one drug approval, activated protein C (Xigris), and an off-label indication for hydrocortisone.

Now, two studies have identified new targets that focus on, and reveal more about, different mechanisms in sepsis. The first, from Tracey and colleagues in Proceedings of the National Academy of Sciences, describes how antagonists of the transcription factor high-mobility group box 1 (HMGB1) can target mediators of the cytokine response of host cells to bacterial toxins. What makes this different from other early inflammatory mediator targets that have been identified, such as tumour-necrosis factor (TNF) and interleukin-1 $\beta$ (IL-1 $\beta$ ), is that HMGB1 has a more delayed kinetic profile: the acute kinetics of TNF and IL- $1 \beta$ provide too narrow a therapeutic window for inhibitors of these cytokines to be clinically useful.

Using the cecal ligation and puncture (CLP) mouse model, regarded as the most clinically relevant model for sepsis, the authors showed that serum HMGB1 levels are increased significantly 18 hours post CLP, corresponding with the clinical development of the disorder. Antibodies to HMGB1 administered 24 hours after surgery increased survival significantly, which is striking as this is the first cytokine-based therapy that is effective when administered more than 8 hours after CLP, and antibodies to TNF worsened outcome in the CLP model. Structure-function studies showed that a domain called the HMGB1 A box inhibits HMGB1 activity, and animals treated with this HMGB1 antagonist were protected from organ injury and were conferred with lasting protection against lethality.

The second study, published in Nature Medicine, shows how the endogenous lysophospholipid lysophosphatidylcholine (LPC) treats sepsis in CLP, as well as in a model involving intraperitoneal injection of Escherichia coli, through a novel mode of enhancing bactericidal activity. The approach of Song and colleagues was

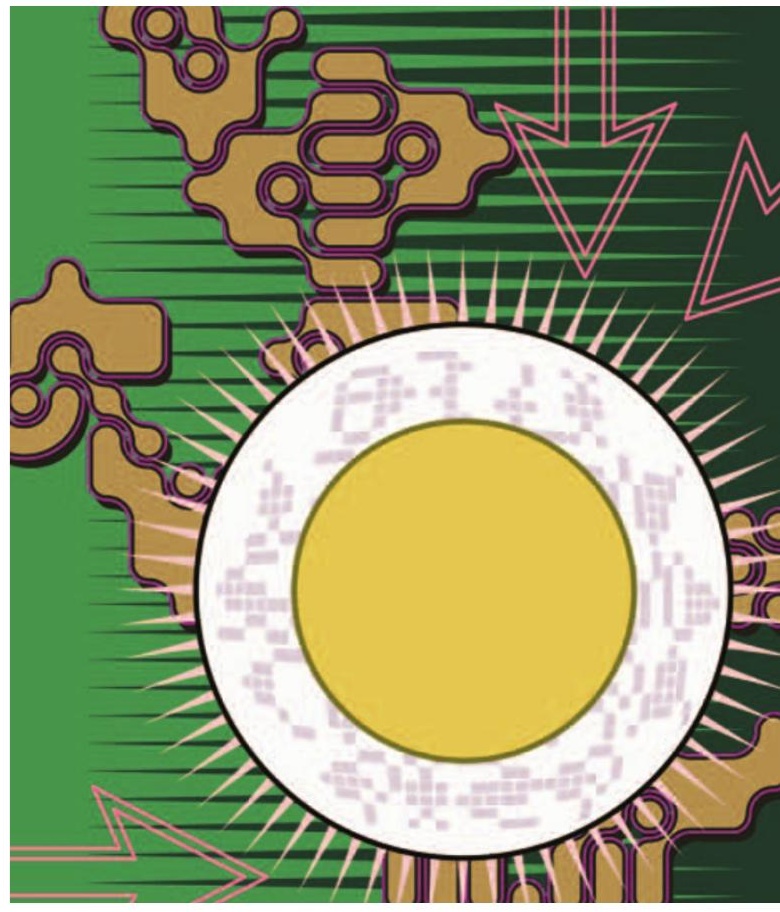

predicated on the notion that defects in neutrophils, monocytes and other immune cells influence sepsis-associated mortality, and looked at LPC, a major component of oxidized lowdensity lipoprotein, which stimulates many immune cells in vitro.

They found that LPC was a potent antiseptic agent in preclinical models, and had a wide therapeutic window of 10-24 hours post-CLP surgery. This antiseptic effect seems to work through two mechanisms: enhancement of bacterial elimination and inhibition of the actions of the bacterial endotoxin lipopolysaccharide. In vitro, LPC increased the bactericidal activity by enhancing $\mathrm{H}_{2} \mathrm{O}_{2}$ production in neutrophils that ingested E. coli, but did not seem to work on any other immune cell. In the sepsis models, antibodies to the LPC receptor G2A inhibited these activities and increased mortality.

Given that so many potential sepsis therapeutics have shown only modest clinical efficacy despite the dramatic effects observed in preclinical models, the future of these targets is by no means certain. But both targets have the advantage of being complementary to current approaches and, in the case of HMGB1, this could allow the clinical subtyping of this complex and heterogeneous disorder.

Simon Frantz

B) References and links

ORIGINAL RESEARCH PAPERS Yang, $\mathrm{H}$. et al. Reversing

established sepsis with antagonists of endogenous high-mobility group box 1. Proc. Nat/ Acad. Sci. USA 101, 296-301 (2004) | Yan, J.-J. et al. Therapeutic effects of lysophosphatidylcholine in experimental sepsis. Nature Med. 11 Jan 2004 doi:10.1038/nm989

FURTHER READING Marshall, J. C. Such stuff as dreams are made on: mediator-directed therapy in sepsis. Nature Rev. Drug Discov. 2 , 391-405 (2003) 